\title{
Embarazo ectópico post-esterilización
}

\author{
Alvaro Monterrosa Castro*
}

\begin{abstract}
RESUMEN: La esterilización femenina es una de las formas más eficaces de anticoncepción. Diversos métodos —a cielo abierto y vía laparoscópica-, han sido difundidos, teniendo todos ellos tasas bajas de falla. Si una paciente resulta embarazada teniendo esterilización previa, tendrá un aumento en las probabilidades de que ese embarazo sea extrauterino. Uno de cada seis embarazos post-esterilización será ectópico. Se presenta el caso de un ectópico no roto post-esterilización con anillos de Yoon. Se presentan las diferentes teorías existentes que explican los mecanismos por los cuales se produce embarazo ectópico posterior a planificación quirúrgica femenina. La incidencia del embarazo ectópico se ha incrementado notablemente, considerándose un caso por cada 1000 embarazos intrauterinos. Las nuevas pruebas sensibles de gestación, la ecografía y la laparoscopia son métodos que se complementan entre sí, ante la sospecha de embarazo ectópico. La laparoscopia es un procedimiento óptimo para diagnóstico de embarazo ectópico roto o no roto. Tiene tasas de falso positivo: $1,6 \%$ y falso negativo de $0.1 \%$.
\end{abstract}

PALABRAS CLAVES: Embarazo extrauterino, embarazo ectópico, esterilización quirúrgica, embarazo post esterilización, planificación familiar.

SUMMARY: Women sterilization is one of the most efective ways of birth control. Different methods at open shy and via laparoscopic have been spread with a low rate of failure. If a patient gets pregnant having been sterilizing, the changes of an extra-uterine pregnancy will increade. One out of six post sterilization pregnancies will be ectopic. There exist the case of a non broken post-sterilization ectopic with yoon rings. There appear different theories which explain the mechanisment why ectopic pregnancy takes place after surgery sterilization a woman. The incidence of ectopic pregnancy has increased noticeably, estimating one case in every thousand intra-uterine pregnancy. The new sensorial testing in pregnancy, ultrasonography and laparoscopic complement each other, in case of a suspicious ectopic pregnancy. Laparoscopic is an excellent procedure to diagnostic an ectopic pregnancy broken or un broken, with a rate of false positive $1.6 \%$ and false negative $0.1 \%$.

KEY WORDS: Extra-uterine pregnancy, ectopic pregnancy, surgical sterilization, post esterilization pregnancy, birth control.

\section{Introducción}

Diversos autores sostienen que la incidencia del embarazo ectópico se ha incrementado notablemente en las últimas décadas (1-6). Taylor (3) afirma que en los pasados diez años la tasa cambió de un caso por cada 123 recién nacidos vivos, a un ectópico por cada 36 nacimientos. Flett et al. (5) informan que en el período de 1950 a 1973, se presentaba un embarazo ectópico por cada 1000 gestaciones intrauterinas, incrementándose a 6 por cada 1000 en el intervalo 1980 a 1984 . A pesar del uso de muy diversos denominadores, que han tornado difíciles las comparaciones (1-2), todas las referencias siguen siendo insatisfactorias para establecer las tasas de riesgo de la mujer al embarazo ectópico (5).

Aunque las razones reales que sustentan el aumento en la incidencia del embarazo extrauterino son inciertas (5), el aumento en la rata de embarazos ectópicos es atribuible a tres factores: incremento en la prevalencia de infecciones tubáricas y enfermedades sexualmente transmitidas, mayor

* Ginecólogo. Laparoscopista. Clínica Profamilia. Cartagena-Colombia. ejecución de procedimientos quirúrgicos relacionados con el manejo de la reproducción y el desarrollo de una cada vez más eficiente metodología diagnóstica $(1,3,5,7)$.

Es posible realizar el diagnóstico temprano de la gestación ectópica, gracias a la disponibilidad de sensibles pruebas para evaluar muy bajos niveles de gonadotropina coriónica, $(3,8-9)$ y a la aplicación de métodos tecnológicos invasivos y no invasivos (3, 8-9). Más sin embargo, para diagnosticar el embarazo ectópico, sobre todo no roto, se amerita un elevado índice de sospecha (1), siendo muy importante la elaboración detallada de la historia clínica, sin olvidar investigar los diversos factores de riesgo para la patología (5). La mayor y pronta precisión en el diagnóstico han permitido reducir las alarmantes tasas de morbilidad y mortalidad materna (11-12). El diagnóstico antes de la ruptura es un desafío médico, que brinda condiciones para adelantar tratamientos conservadores. Se han utilizado: la administración de metotrexate $(3,13-14)$, y la implementación de técnicas quirúrgicas a cielo abierto o laparoscópicas, para punciones, aspiraciones, salpingostomías lineales o aplicación local de metotrexate $(8,10,15-17)$, permitiendo conservar la integridad anatómica de la trompa, preservando la oportunidad reproductiva $(3,7)$. Mashiach et al. (7) han informado casos de embarazos tubáricos tempranos no 
rotos, resueltos espontáneamente por absorción del tejido gestacional, en pacientes que habían sido manejadas sólo con observación. Los mismos autores (7) afirman, que se puede estar sobre diagnosticando y sobre tratando el embarazo tubárico. Las nuevas tecnologías diagnósticas, al decir de Taylor (3), pueden estar incrementando iatrogénicamente las tasas de embarazo ectópico, al incluir casos que posiblemente se resolverían sin intervención. Es preciso recordar que generalmente, cuando el embarazo ectópico experimenta ruptura, estaremos ante una emergencia - algunas veces catastrófica-, en la cual retrasar el diagnóstico y el manejo puede conllevar amenazas para la vida de la paciente $(5)$.

La causa concreta del embarazo ectópico es desconocida (18), estando la alteración en los mecanismos fisiológicos de transporte tubárico fuertemente incriminados (2). Las trompas de falopio no son un simple conducto para transporte; son el sitio de capacitación de los espermatozoides, captura y fecundación del oocito, proporcionando sostén y nutrición al óvulo fecundado en su ruta hacia la cavidad uterina (18). Los mecanismos propulsores tubáricos, o sea: los movimientos de las cilias y las contracciones de la musculatura de la pared, se pueden alterar cuando la paciente ha padecido episodios inflamatorios pelvianos (1, 44), ha sido sometida a manejo quirúrgico con miras a restablecer la fertilidad, o se ha recurrido a procedimientos operatorios de esterilización $(3,5-6,19)$. Los dispositivos intrauterinos también han sido considerados factores de riesgo, en relación con el embarazo ectópico $(1-2,6)$. Alteraciones $\mathrm{y}$ disfunciones endocrinas $\mathrm{u}$ ovulatorias, embriogénesis anormal y transmigración abdominal de óvulos y espermatozoides, han sido mecanismos involucrados al intentar explicar las causales del embarazo extrauterino $(2,5,20-21)$.

\section{Historia clínica}

H.C. 1231765 de la Clínica Profamilia, Cartagena, Colombia. Paciente de 36 años de edad, que el 6 de diciembre de 1990 consultó por presentar flujo fétido y amarillento de cinco meses de evolución, que no había mejorado a diversos tratamientos médicos. Su última menstruación fue el 24 de octubre de 1990 y no había dado importancia a su atraso menstrual. Menarquia: 14 años. G:3, P:3, A:0. Esterilización por lapacoscopia (anillos de Yoon) en enero de 1989. No presentaba antecedentes quirúrgicos ni médicos de interés.

Signos vitales normales. Examen físico general normal. Abdomen blando, sin masas ni dolor. Especuloscopia: flujo abundante, amarillo, espeso, no fétido. Cuello sano con escasa secreción sanguinolenta proveniente de cavidad uterina. Al tacto bimanual: útero en AVF con cuello duro, algo aumentado de tamaño. Ausencia de dolor en anexos y no se determinaron masas para-uterinas. Ante la sospecha de gestación, se ordenó prueba de embarazo en sangre, la cual fue realizada el 9 de diciembre, con resultado positivo. El 12 de diciembre presentó sangrado escaso, súbito y momentáneo acompañado de la expulsión de una masa carnosa, la cual fue traída a la clínica y considerada macroscópicamente como molde endometrial. Se sospecha emba- razo extrauterino y se realiza ultrasonografía pélvica transabdominal (transductor lineal de 35) observándose útero de $100 \times 51 \times 53 \mathrm{~mm}$, de contornos lisos, sin saco gestacional en su interior. En anexo izquierdo se observó una zona anecoica ovalada de $30 \mathrm{~mm}$ de diámetro mayor. Anexo derecho normal. Ausencia de líquido en el fondo de saco de Douglas. Para corroborar la impresión de embarazo ectópico no roto de trompa izquierda, se realizó laparoscopia diagnóstica. Se visualizó útero normal y ovarios normales. Trompa derecha normal con anillo de Yoon en tercio medio. Trompa izquierda con anillo de Yoon en tercio medio, y en el istmo una estructura redondeada y violácea de aproximadamente dos o tres centímetros de diámetro que distendía el mesosalpinx. Con diagnóstico definitivo de embarazo ectópico no roto de trompa izquierda, se realizó laparotomía para salpinguectomía total. El estudio anatomopatológico de la trompa extirpada, evidenció la presencia de vellosidades coriónicas. La valoración histológica de la masa expulsada por vía vaginal, fue informada como molde decidual, aseverando el patólogo la ausencia de vellosidades coriales. La paciente evolucionó favorablemente.

\section{Comentarios}

La esterilización femenina representa un significativo beneficio para la salud, particularmente en países con altas tasas de mortalidad materna asociada a la gestación. David Grimes et. al. (22) basados en estudios adelantados en Bangladesh, estiman que por cada 100.000 esterilizaciones se previenen más de 1000 muertes maternas. La esterilización femenina es una de las formas más eficaces de anticoncepción (23). La minilaparotomía para ligadura tubárica se desarrolló en el decenio de los setenta, y si bien es probablemente el método más seguro de esterilización femenina (24-25), la popularidad de la esterilización laparoscópica se ha incrementado en todo el mundo (2527). Esta difusión se ha facilitado por sus múltiples ventajas, y sobre todo, por la poca frecuencia de complicaciones (12, 26). Khadndwala (26) presenta informes de Philips, quien en 77.103 esterilizaciones por laparoscopia, reporta morbilidad del $0.27 \%$ y mortalidad de $4 / 100.000$ pacientes. El mismo autor (26) anota que Chamberlain en 29.661 esterilizaciones encontró morbilidad del $3.6 \%$ y mortalidad de 10/100.000. Las complicaciones por el método laparoscópico, serán directamente proporcionales a la experiencia del operador y al cuidado en la aplicación de los pasos a seguir $(12,23)$.

Diversas técnicas para esterilización laparoscópica se han estandarizado: Electrocauterización unipolar y bipolar, endocoagulación, clips de Hulka y anillo o banda de Yoon (12, 19, 25-26, 28). La aplicación de anillos de Yoon técnica oclusiva-, ha reemplazado paulatinamente a los métodos destructivos: electrocoagulación y endocoagulación, por presentar éstos últimos más riesgos.

Las tasas de falla, embarazos, son aceptablemente bajas para todos los métodos $(5,26)$. Menos de una mujer por cada 100 esterilizadas quedará embarazada en los dos años posteriores a la intervención quirúrgica (12). Lassner et al. (23) en un estudio en 13.423 mujeres, determinan que a los 6 meses post-esterilización la tasa estimada es de 0.28 
embarazos por 100 esterilizariones, para ascender a 0.54 hacia los doce meses, 0.87 a los 24 meses, 0.99 a los 36 meses y 1.04 a los 48 meses. La probabilidad de falla en la esterilización, se duplicará del primero al cuarto año posterior a la cirugía (23).

Si una paciente resulta embarazada teniendo esterilización previa, tendrá un aumento en las probabilidades de que ese embarazo sea ectópico (3). Frank de Stefano et al. (29) citan estudios que indican que uno de cada seis embarazos que ocurren después de esterilización, es ectópico. El incremento en las probabilidades para el embarazo extrauterino será del 15 al 20\%, siendo mayor si la esterilización se adelantó por cauterización tubárica $(1,5,8,29$ 30 ).

Mc Causland (31) estudió piezas de histerectomía, y evidenció que las pacientes previamente sometidas a ligadura tubárica con electrocauterización tenían un $75 \%$ de probabilidades de tener fístula tubo-peritoneal. Este mismo autor (30) sostiene que posterior a la cauterización tubárica, se puede producir una activación del epitelio tubárico, endosalpingiosis, invadiendo entonces el miosalpingx, la serosa tubárica o el miometrio: formándose verdaderas fístulas tubo-peritoneales o útero-peritoneales. Rusell (18) asevera que cuanto mayor sea el daño tubárico circundante, mayor será la posibilidad de endosalpingiosis, la formación de fístulas y la oportunidad de embarazo extrauterino.

La aplicación laparoscópica de electrocauterización bipolar (corriente eléctrica de alta frecuencia) y la endocoagulación (calor); tienen mínimas dificultades operatorias y son técnicas de gran valor en presencia de adherencias pélvicas y patologías tubáricas (26). Las complicaciones quirúrgicas son considerablemente poco frecuentes, pero pueden llegar a ser serias, generalmente quemaduras de asas intestinales o vejiga. Riedel (32) en el XIIth World congress on fertility and sterility presentó tasas de quemadura con bipolar del $0.10 \%$ y $0.02 \%$ con el endocoagulador. Con estos dos métodos las tasas de embarazos son sumamente bajas, $0.4 \%$ para bipolar y $0.11 \%$ para endocoagulación, pero con un riesgo alto de embarazo ectópico $-35 \%$ y $27 \%$ respectivamente (32)-, debido a alta oportunidad de formación de fístulas tubo-peritoneales (33). Kleppinger (34) considera que con electrocauterización, las tasas de falla son del $0.25 \%$, siendo el $65 \%$ de ellas, embarazos ectópicos. Sugiere que para reducir el riesgo de fístula tubo-peritoneal, debe evitarse la cauterización tubárica a menos de $2.5 \mathrm{cms}$ del cuerno uterino. Con ambos métodos de cauterización se destruyen grandes segmentos tubáricos $(26,28)$. Rusell (18) concuerda con lo afirmado por los autores anteriores, y podemos dejar sentado que los procedimientos que incluyen destrucción tubárica extensa, se correlacionan con elevadas tasas de embarazos extrauterinos. Igual sostiene Mc Clausland (30).

La electrocauterización unipolar ha sido abandonada, por la gran destrucción tubárica que ocasiona y sobre todo, por el elevado riesgo de producir quemaduras a distancia en otros órganos $(12,28)$.

El anillo tubárico o banda de silastic o anillo de Yoon, conocido comercialmente como Falope Ring, es el dispositivo de oclusión tubárico más utilizado (12), desarrollado por In Bae Yoon en 1973 en Johns Hopkins University (35). Se aplica en la unión de la ampolla y el istmo, a tres centímetros del cuerno uterino. Con el anillo de Yoon suele destruirse entre uno y tres centímetros de trompa $(12,28)$. Si bien el anillo de Yoon puede aplicarse con facilidad en la mayoría de los casos, suelen presentarse dificultades o imposibilidad, en trompas gruesas, vascularizadas, edematosas o con adherencias $(12,35)$. Poliakoff et al. (36) encontraron tasas del $3.7 \%$ para complicaciones intraoperatorias al aplicar anillos de Yoon, estando dadas generalmente por ruptura tubárica y trauma al mesosalpinx.

Metz y Mastroianni (20) afirman, aunque sea difícil demostrar, que la existencia de fístulas y la recanalización tubárica espontánea, fístulas tubo-tubáricas, permiten transmigración de espermatozoides, óvulos o ambos. En el caso presentado, la ubicación del ectópico y la disección del mesosalpingx, nos permiten sospechar la formación de una fístula por debajo del anillo de Yoon que facilitó la fecundación, y ante una inadecuada actividad propulsora permitió el desarrollo por fuera de la cavidad uterina (33).

La oclusión incompleta de la luz tubárica (21) al colocar el anillo de Yoon, sea por trompas edematosas y gruesas, o por mala técnica o error del operador, puede ser otra explicación para la falla del método, obteniéndose generalmente producto intrauterino $(12,24,36)$. Trias et al. (24) por un muy amplio estudio realizado en Clínica Profamilia de Bogotá, Colombia, determinan que en pacientes que se embarazan posterior a la esterilización, en el $19 \%$ de los casos existe error en la técnica operatoria. Una trompa disfuncional o traumatizada por un anillo mal colocado, puede servir de base para un posterior embarazo tubárico. Iype (37) presenta tres casos de embarazo tubárico post aplicación de anillo de Yoon.

Rusell (18) asevera que en general las fallas en las técnicas laparoscópicas de esterilización oclusivas (anillos, clips), suelen ser embarazos intrauterinos. Poliakoff et al. (36), para estos métodos establece tasas de falla del $0.25 \%$ y no informa del riesgo para embarazo ectópico. Chi y Col. (38) anotan cifras muy cercanas al $0.33 \%$ para anillos de Yoon y de $0.5 \%$ para clips de Hulka. Suporn Koetsawang et al. (28) fijan tasa de falla del $0.1 \%$ y $0.5 \%$ respectivamente, aseverando que con el clips de Hulka se destruye menos de un centímetro de trompa. En 1972 F. Hulka y George Clemens dieron a conocer el modelo original (12). El clips de Hulka consiste en dos brazos de plástico con dientes que se cierran entre sí. Los brazos se mantienen unidos por un resorte de acero inoxidable, que es empujado hacia adelante para trabar el clips y fijarlo en su posición en la trompa (12, 39). La esterilización fallida es un factor muy relacionado con el embarazo ectópico $(5,40)$. El estudio y seguimiento de la relación entre esterilización y un subsecuente embarazo ectópico es difícil de realizar (5). Mac Causland (30) anota que de los embarazos ocurridos después de esterilización no laparoscópica, el 12,3\% fueron ectópicos, rata que se aumenta al $51 \%$ si fue por cauterización laparoscópica. Por su parte Flett et al. (5) en su revisión, de 3.104 esterilizaciones por laparotomía postparto encontraron seis ectópicos, fijando una tasa de 1,9/1.000 esterilizaciones. Seis ectópicos en 2.500 minilaparotomías para una rata de $2,4 / 1.000$. Un total de 23 ectópicos sucedieron en 4.261 esterilizaciones por vía laparoscópica, estableciendo una rata de 5,4/1.000. De una manera global los autores (5) establecen una prevalencia de embarazo ectópico en muje- 
res esterilizadas por todos los métodos del 3,5/1.000, y citan estudios de Tatum y Schmidt que encontraron, al utilizar clips, un ectópico por cada 1.000 esterilizaciones. Igual tasa determinó Mac Causland (30) en esterilizaciones no laparoscópicas. Flett et al. (5) en su estudio demostraron que aproximadamente 0.35 embarazos extra uterinos ocurren por 1.000 años-mujer esterilizadas.

Se debe proceder con cautela ante la paciente con antecedente de esterilización que presenta dolor abdominal. No admitir falsa sensación de seguridad por el hecho de tener historia de esterilización previa (51). No existen signos ni síntomas patognomónicos de embarazo tubárico (30). Como en el caso presentado, puede la paciente estar totalmente asintomática mientras esté el ectópico sin romperse. El útero llegará a crecer como si el embarazo fuese intrauterino en el $26 \%$ de los casos. En el $70 \%$ conservará sus dimensiones normales (40). El $75 \%$ de las pacientes relatarán historia de amenorrea o irregularidad menstrual en los ciclos precedentes (2). El 50\% presentarán manchado vaginal escaso y menos de la mitad tendrán masa anexial palpable (2). Sólo el $20 \%$ referirán síntomas relacionados con el inicio del embarazo. El 10\% expulsarán masa por los genitales externos, que corresponderá al molde endometrial. Romero et al. (41) informan que en el $45 \%$ de las pacientes con hemoperitoneo por embarazo ectópico roto no presentarán signos de irritación peritoneal. Sólo con un alto índice de sospecha (1) y siendo notablemente acucioso, se puede pensar en el embarazo ectópico no roto. Para entonces deben realizarse estudios para definir la presencia o no de la patología.

En el embarazo ectópico se producen menores niveles de Gonadotropina coriónica, que en el embarazo normal (2, 42), por tal razón las determinaciones ordinarias suelen ser negativas. Nuevas pruebas, mucho más sensibles han sido introducidas en los últimos años. Estudios en suero por métodos ELISA y RIA tienen sensibilidad en embarazo ectópico cercanas al $100 \%$.

El ultrasonido está adquiriendo una gran importancia en la evaluación de la paciente en quien se sospecha embarazo extra uterino. Ya que se sospecha embarazo ectópico más a menudo de lo que ocurre (43), el método diagnóstico que permita definir con claridad su presencia será sumamente valioso $(2,11)$. El papel del ultrasonido es indicar aquellas pacientes con alta probabilidad de embarazo tubárico, en quienes estará indicada la laparoscopia (11). La adición del ultrasonido como método auxiliar no invasivo en el estudio del embarazo ectópico, ha proporcionado precisión en el diagnóstico pre-operatorio, disminuyendo el número de laparoscopias innecesarias. Examen con ultrasonido en el momento adecuado, en la paciente de riesgo, permite además del diagnóstico temprano, disminución en la morbilidad (11).

El examen bajo anestesia ha caído en desuso. Los embarazos ectópicos tempranos, no rotos, por lo general son pequeños para ser palpados y un tacto vigoroso podría romperlos. Si la paciente ha de recibir anestesia, es correcto seleccionar un método diagnóstico más preciso y exacto. Ese método es la laparoscopia. Esposito (43) presenta los resultados de 1.264 pacientes con sospecha de embarazo ectópico evaluadas por laparoscopia. En el $31 \%$ se confirmó la existencia del ectópico. Sólo en el $1 \%$ fue imposible la valoración por la existencia de adherencias múltiples. En el $50 \%$ se evitó realizar laparotomía. Esposito (43) ratifica el gran valor del estudio laparoscópico al fijar tasas de falsos positivos del $1.6 \%$ y falsos negativos del $0.1 \%$. La laparoscopia es un método invasivo óptimo para diagnosticar el embarazo ectópico roto o no roto (12).

Las nuevas pruebas sensibles de embarazo, la evaluación eco-sonográfica pélvica transvaginal y la laparoscopia, son estudios que se complementan entre sí $(9-10,45)$.

Si bien algunos autores (29) sostienen que el incremento en la popularidad de la esterilización, no ha contribuido a elevar la incidencia del embarazo ectópico, hay que aseverar que la esterilización tubárica sí es un factor que aumenta el riesgo para esta patología (2). Por tal razón, el embarazo ectópico es una condición a tener en cuenta en la mujer con esterilización previa, en quien es posible la existencia de gestación.

\section{BIBLIOGRAFIA}

1 Franks AL., Beral V., Cates Jr. W. et al. Contraception and ectopic pregnancy risk. Am. J. Obstet. Gynecol. 1990; 163(4): part 1: 11201123.

2. Stabile I., Grudzinskas JG. Ectopic pregnancy: A review of incidence, etilogy and diagnostic aspects. Obstet. Gynecol. surv. 1990; 45(6): 335-347.

3. Taylor RN. Ectopic pregnancy and reproductive technology Jama 1988; 259(12): 1862-1863.

4. Loffer FD. The increasing problem of ectopic pregnancies and its impact on patients and phisicians. J. Reprod. Med. 1986; 31: 74-77.

5. Flett GM., Urquhart DR., Fraser C. et al. Ectopic pregnancy in Aberdeen 1950-1985. Br. J. Obstet. Gynecol. 1988; 95: 740-746.

6. Marchbanks PA., Annegers JF., Coulam CB. et al. Risk factors for ectopic pregnancy. A population-based study. Jama 1988; 259(12): 1823-1827.
7. Mashiach S., Carp HJA., Ser DM. Nonoperative management of ectopic pregnancy. J. Reprod. Med. 1982; 27: 127-132.

8. Hill GA., Cartwrighy PS., Herber CM. Retained trophoblast after conservative management of ectopic pregnancy. J. Reprod. Med. 1990; 35(4): 415-417.

9. Sepalla M., Purhonen M. Uso de HCG y otras proteínas del embarazo en el diagnóstico del embarazo ectópico. Clin. Obstet. Ginecol. 1987; 1: $143-148$.

10. Johns A. Consider laparoscopy for an ectopic gestacion. Contemporary Ob/Gyn 1989; 34 ( $\left.\operatorname{spec} N^{\circ}\right)$ 67-72.

11. DeCrespingy LCh. Aplicaciones del ultrasonido en el embarazo ectópico. Clin. Obstet. Ginecol. 1987; 1: 131-141.

12. Liskin L., Rinehart W. Minilaparotomy and laparoscopy: safe, effective and widely use. Population report. Serie C-9. Population information program, Baltimore, 1985. 
13. Ory SJ., Villanueva AL., Sand PK. et al. Conservative treatment of ectopic pregnancy with methotrexate. Am. J. Obstet. Gynecol. 1986; 154: 1299-1301.

14. Sauer MV., Gorriel MJ., Rodi IA. et al. Nonsurgical management of unruptured ectopic pregnancy: an extended clinical trial. Fertil Steril 1987; 48: 752-755.

15. Cartwright PS., Herbert CM III., Maxson WS. Operative laparoscopy for the management of tubal pregnancy. J. Reprod. Med. 1986; 31 589-571.

16. Pouly JL., Mahnes H., Mage G. et al. Conservative laparoscopic treatment of 321 ectopic pregnancies. Fertil Steril 1986; 46: 10931095.

17. Zakut H., Sadan O. Katz A. et al. Management of tubal pregnancy with methotrexate. Br. J. Obstet. Gynecol. 1989; 96(6): 725-728.

18. Russell JB. The etiology of ectopic pregnancy. Clin. Obstet. and Gynecol. 1987; 30(1): 187-190.

19. Pellicer A., Serra V. Femele sterilization using tubal coagulation. Adv contracep delivery sistems 1988; 4(4): 349-367.

20. Metz KG., Mastroianni L. Tubal pregnancy subsequent to transperitoneal migration of spermatozoa. Obstet. Gynecol. Surv. 1979; 34: 554-559.

21. Soderstrom RM. Sterilization failures and their causes. Am. J. Obstet. Gynecol. 1985; 152(4): 395-403.

22. Grimes DA., Peterson HB., Rosemberg MJ. et al. Sterilizations atributable deaths in Bangladesh. Int. J. Gynecol. Obstet. 1982; 20(2): 149-154.

23. Lassner KJ. et al. Analysis of sterilization failure in Brazil. Int. J. Gynecol. Obstet. 1988; 27(3): 255-258.

24. Trias M., Anderson JE., Ojeda G. et al. A life table analysis of sterilization failure: Date from the Profamilia Clinic, Bogotá, Colombia. Int. J. Gynecol. Obstet. 1987; 25(3): 235-240.

25. Bhiwandiwala PP., Numford SD., Feldblum PJ. A comparison of different laparoscopic sterilization oclusion techniques in 24439 procedures. Am. J. Obstet. Gynecol. 1982; 144(3): 319-331.

26. Khandwala SD. Laparoscopic sterilization. A comparison of curren techniques. J. Reprod. Med. 1988; 33(5): 463-466.

27. Rioux JE., Soderstrom RM. Sterilization revisited. Contemporary Ob/ Gyn 1987; 30(2): 80-104.

28. Koetsawang S., Gates DS., Suwanichati S. et al. Long term follow-up of laparoscopic sterilization by electro-coagulation, the Hulka Clip and the tubal ring. Contraception 1990; 41(1): 9-18.
29. DeStefano F., Peterson HB., Layde PM. et al. Risk of ectopic pregnancy following tubal sterilization. Obstet. Gynecol. 1982; 60(3): 326-330

30. Mc Causland A. A hight rate of ectopic pregnancy following laparoscopic tubal coagulation failures. Am. J. Obstet. Gynecol. 1980; 163: 97-101.

31. Mc Causland A. Endosalpingosis (endosalpingoblastosis) following laparoscopic tubal coagulation as an etiologic factor of ectopic pregnancy. Am. J. Obstet. Gynecol. 1982; 143: 17-22.

32. Riedel HH., Semm K. German pelviscopic statistics for the years 1978-1982. In Handbook of abstracts of XIIth world congress on fertility and sterility, Singapore. 1986; 415.

33. Stock RJ., Nelson KJ. Ectopic pregnancy sussequent to sterility histologic evaluation and clinical implication Fertil Steril 1984;42(2): 211-215.

34. Kleppinger RK. Laparoscopy in tubal sterilization. In: Garcia CR., Mikula JJ., Rosemblum NG. Current Therapy in surgical gynecology. Philadelphia. 1987; 83-86.

35. Yoon IB. Silicone ring. IN: Phillips Jm. Laparoscopy. Baltimore, Williams \& Wilkins, 1977; 174-178.

36. Poliakoff SR., Yoon IB., King TM. A four year experience with the Yoon ring. IN: Phillips JM., Duoney CC. Endoscopy in Gynecology. American Association of ginecologics laparoscopist 1978; 197-207.

37. Iype E., Nair VR., Girija B. Tubal pregnancy following laparoscopic sterilization. Asia-Oceania J. Obstet. Gynecol. 1987; 13(2): 201-203.

38. Chi IC., Potts M., Wilkens L. Rare events associated with tubal sterilizations: an international experience. Obstet. Gynecol., Surv. 1986; 41(1): 7-12.

39. Hulka JF., Omran K., Lieberman BA. et al. Laparoscopic sterilization with the spring clip: instrumentation development and current clinical experience. Am. J. Obstet. Gynecol. 1979; 135(8): 1016-1020.

40. Brenner PF., Roy S., Mishell DR. Ectopic pregnancy: a study of 300 consecutives surgically treated cases. JAMA 1980; 243: 673-574.

41. Romero R., Copel MA., Kadar N. et al. Value of culdocentesis in the diagnosis of ectopic pregnancy. Obstet. Gynecol. 1985; 65: 619-624.

42. Ackerman R., Deutsche S., Krumholz B. Levels of HCG in unrupture and ruptured ectopic pregnancy. Obstet. Gynecol. 1982; 60: 13-16.

43. Esposito JM. Ectopic pregnancy. The laparoscope as a diagnostic aid J. Reprod. Med. 1980; 35: 17-22.

44. Muydelr. Ectopic Pregnancy in Zambabwe. Int. J. Gynecol. Obstet. 1991; 35: 55-60.

45. ACOG. Technical bulletin Ectopic Pregnancy. Int. J. Gynecol. Obstet. 1992; 37: 213-219.

\section{EVENTOS}

II CURSO DE ACTUALIZACION EN MENOPAUSIA Y MEDICINA GENERAL Agosto 13 y 14 en Barranquilla. Informes (958) 568710

FIGO 94 14 WORLD CONGRESS OF GINECOLOGY AND OBSTETRICS Septembre 24 - 30/94 Montreal - Canadá

\section{UNIVERSIDAD DE ANTIOQUIA FACULTAD DE MEDICINA INVITAN TOPICOS SELECTOS DE INFECTOLOGIA 1994}

16 y 17 de septiembre de 1994 Teatro Pablo Tobón Uribe - Medellín

Informes: Depto. de Microbiología y Parasitología Tel.: (942) 635411 Medellín 